Check for updates

Cite this: RSC Adv., 2017, 7, 37276

Received 16th June 2017

Accepted 17th July 2017

DOI: 10.1039/c7ra06716j

rsc.li/rsc-advances

\section{In situ synthesis and electromagnetic wave absorbing properties of sandwich microstructured graphene/La-doped barium ferrite nanocomposite}

\author{
Tingkai Zhao, (D) * Wenbo Jin, ${ }^{*}$ Yixue Wang, Xianglin Ji, Huibo Yan, Chuanyin Xiong, \\ Xufei Lou, Alei Dang, Hao Li and Tiehu Li
}

\begin{abstract}
The development of high reflection loss and broad frequency bandwidth for electromagnetic wave absorbing materials has been pursued for a long time. Constructing a rational microstructure of an absorber will have significant impact on reflection loss increase and frequency bandwidth broadening. Herein, we successfully prepare a sandwich microstructured graphene/ $\mathrm{BaFe}_{12} \mathrm{O}_{19}$ nanocomposite by an in situ auto-combustion method. Compared to pure $\mathrm{BaFe}_{12} \mathrm{O}_{19}$, the sandwich microstructured graphene/ $\mathrm{BaFe}_{12} \mathrm{O}_{19}$ showed better electromagnetic wave absorbing properties. Furthermore, the sandwich microstructured graphene/ $\mathrm{Ba}_{0.8} \mathrm{La}_{0.2} \mathrm{Fe}_{12} \mathrm{O}_{19}$ nanocomposite was prepared with La-doped $\mathrm{BaFe}_{12} \mathrm{O}_{19}$ using the same method. The obtained graphene/Ba.8 $\mathrm{La}_{0.2} \mathrm{Fe}_{12} \mathrm{O}_{19}$ nanocomposite exhibited a saturation magnetization of $26.55 \mathrm{emu} \mathrm{g}^{-1}$ at room temperature and exhibited excellent magnetic performance. The maximum reflection loss of the sandwich microstructured graphene/ $/ \mathrm{Ba}_{0.8} \mathrm{La}_{0.2} \mathrm{Fe}_{12} \mathrm{O}_{19}$ nanocomposite with a thickness of $1 \mathrm{~mm}$ could reach up to $-40.26 \mathrm{~dB}$, and a frequency bandwidth value below $-10 \mathrm{~dB}$ was observed up to $3.87 \mathrm{GHz}$ within the frequency range of $2-18 \mathrm{GHz}$.
\end{abstract}

\section{Introduction}

With the rapid development of wireless communication technology, electromagnetic wave (EMW) pollution has become a serious environmental problem. High density of electromagnetic waves has a certain impact on human health, particularly in children and the elderly. ${ }^{\mathbf{1}}$ Moreover, in the military field, stealth aircraft, armor and warships show greater viability than traditional equipment. To solve the EMW pollution problem and improve viability of military equipment, considerable interest has been focused to EMW absorbing materials with higher efficiency and wider bandwidths. ${ }^{2,3}$

Graphene, two-dimensional planar sheets composed of $\mathrm{sp}^{2}$ bonded carbon atoms have drawn great attention due to their unique topological structures and properties. ${ }^{4}$ To date, several methods of synthesizing graphene have been developed, such as chemical vapor deposition (CVD), mechanical exfoliation and oxidation reduction. Graphene is a single-layer graphite compound with a high specific surface area, high temperature resistance, low weight, excellent electrical conductivity and great potential for application in various fields. ${ }^{5}$ Furthermore, due to its excellent dielectric properties and low density, it is potentially a good EMW absorbing material. However, impedance matching

State Key Laboratory of Solidification Processing, Shaanxi Engineering Laboratory for Graphene New Carbon Materials and Applications, School of Materials Science and Engineering, Northwestern Polytechnical University, Xi'an 710072, China. E-mail: ztk-xjtu@163.com;wenbo_jin@126.com is an essential factor for good EMW absorption; the conductive and electromagnetic parameters of pure graphene prevent it from meeting the requirement of impedance matching, which results in strong reflection but weak absorption. ${ }^{6}$ Moreover, for a high-performance EMW absorbing material, excellent dielectric loss and magnetic loss are two important technical requirements. While graphene has excellent electrical conductivity and shows good dielectric loss, its magnetic loss is poor. Nevertheless, its electromagnetic properties can generally be improved by combining with some magnetic materials, such as magnetic metal powder, ferrite material and some other oxides. It is worth noting that ferrite material is a mature EMW absorbing material with numerous excellent properties. ${ }^{7,8}$ Barium ferrite $\left(\mathrm{BaFe}_{12} \mathrm{O}_{19}\right)$ is one of the most versatile hard magnetic material. It is not only a magnetic medium but also a dielectric medium and exhibits good magnetic loss and dielectric loss. In addition, it has attractive magnetic properties, high Curie temperature, excellent chemical stability and low cost and has been widely used in the field of EMW absorption. Moreover, it can be prepared by a simple auto-combustion method. ${ }^{9}$ Pallab Bhattacharya et al. ${ }^{\mathbf{1 0}}$ have prepared graphene/CuFe ${ }_{10} \mathrm{Al}_{2} \mathrm{O}_{19}$ composite by coprecipitation: in a metal salt solution, the metal oxides were precipitated on the surface of acid-treated graphene. However, this method is insufficient, complex and not environment friendly. Luo Kong et al. ${ }^{11}$ have prepared graphene $/ \alpha-\mathrm{Fe}_{2} \mathrm{O}_{3}$ nanocomposite through a troublesome process. The graphene oxide was dispersed in ethylene glycol and a certain amount of ferric chloride and anhydrous sodium acetate were added. After 
stirring, the mixture was placed in a Teflon-sealed autoclave and maintained at $160{ }^{\circ} \mathrm{C}$ for $12 \mathrm{~h}$, and the product was isolated by magnetic separation. Finally, after washing the product, graphene $/ \alpha-\mathrm{Fe}_{2} \mathrm{O}_{3}$ nanocomposite was obtained. However, this method is too complex and the reaction process is difficult to control. Both methods mentioned above use graphite to prepare graphene, which is then combined with a ferrite material in a metal salt solution. They are troublesome and the ferrite particles are distributed on the surface of graphene unevenly. Furthermore, the EMW absorbing properties of these composites are not satisfactory. However, it should be noted that adjacent graphene layers are bound by weak van der Waals forces in graphite and expanded graphite (EG) has large sheet spacing, which provides a way to prepare graphene by second expansion of EG. ${ }^{12}$ EG has the property of absorbing oil, and the autocombustion precursor of $\mathrm{BaFe}_{12} \mathrm{O}_{19}$ is an oily gel. Furthermore, when the oily gel forms $\mathrm{BaFe}_{12} \mathrm{O}_{19}$, it has a significant volume increase. Therefore, we can take advantage of these properties to in situ prepare a sandwich structure of graphene/ $\mathrm{BaFe}_{12} \mathrm{O}_{19}$ nanocomposite. The oily gel can enter into the layers of EG and the latter will undergo secondary expansion to form graphene by an auto-combustion process using $\mathrm{BaFe}_{12} \mathrm{O}_{19}$ as the precursor. To our knowledge, the preparation and EMW absorbing properties of graphene/ $\mathrm{BaFe}_{12} \mathrm{O}_{19} \quad(\mathrm{G} / \mathrm{BF})$ sandwich structured nanocomposite have not been reported previously. ${ }^{13-17}$

Herein, the graphene/ $\mathrm{BaFe}_{12} \mathrm{O}_{19}$ sandwich structure nanocomposite is successfully prepared using an in situ autocombustion method. Magnetic properties and EMW absorbing properties of the nanocomposite are studied. It is observed that the graphene/ $\mathrm{BaFe}_{12} \mathrm{O}_{19}$ sandwich structured nanocomposite had significantly enhanced EMW absorbing performance compared to pure graphene and pure $\mathrm{BaFe}_{12} \mathrm{O}_{19}$. To obtain excellent EMW absorbing performance, $\mathrm{La}\left(\mathrm{NO}_{3}\right)_{3}$ is added to the $\mathrm{BaFe}_{12} \mathrm{O}_{19}$ precursor solution to prepare graphene/ $\mathrm{Ba}_{0.8} \mathrm{La}_{0.2} \mathrm{Fe}_{12} \mathrm{O}_{19}$ (G/LBF) nanocomposite. The position of partial $\mathrm{Ba}^{2+}$ in the lattice is replaced by $\mathrm{La}^{3+}$ and it can be adjusted and optimized by electromagnetic parameters of the absorbing materials.

\section{Experimental}

\subsection{Preparation of EG}

A certain amount of natural flake graphite was mixed with a mixture of $\mathrm{H}_{2} \mathrm{SO}_{4}$ and $\mathrm{HNO}_{3}$ and stirred at room temperature for $1 \mathrm{~h}$. Then, a small amount of $\mathrm{KMnO}_{4}$ was added to the mixture and stirred for $2 \mathrm{~h}$. The product was washed several times with deionized water until the $\mathrm{pH}$ reached 4-7. After filtration, the black powder was dried in a vacuum oven to obtain graphite intercalated compound. Finally, the graphite intercalated compound was rapidly expanded at $800{ }^{\circ} \mathrm{C}$ in a muffle furnace to obtain EG. ${ }^{18}$

\subsection{In situ synthesis of sandwich microstructured G/BF and G/LBF nanocomposite}

The sandwich microstructured G/BF and G/LBF nanocomposites were synthesized by an in situ auto-combustion

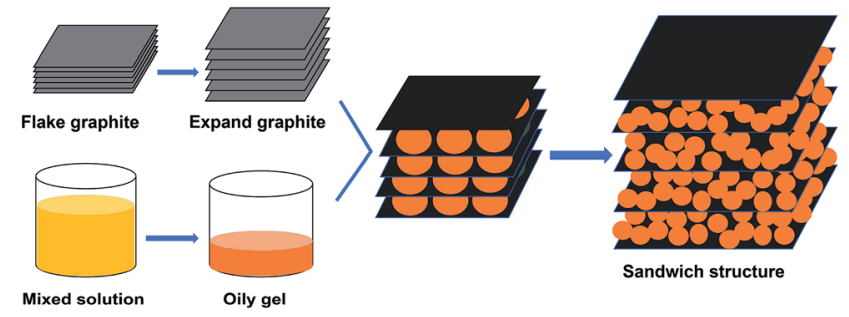

Fig. 1 Schematic of sandwich microstructured $G / B F$ and G/LBF composite synthesis.

method and the schematic is shown in Fig. 1. $\mathrm{Fe}(\mathrm{NO})_{3} \cdot 9 \mathrm{H}_{2} \mathrm{O}$, $\mathrm{Ba}(\mathrm{NO})_{2}, \mathrm{La}\left(\mathrm{NO}_{3}\right)_{3} \cdot 6 \mathrm{H}_{2} \mathrm{O}$ and citric acid were taken as the raw materials. A certain amount of $\mathrm{Fe}(\mathrm{NO})_{3} \cdot 9 \mathrm{H}_{2} \mathrm{O}$ and $\mathrm{Ba}(\mathrm{NO})_{2}$ was dissolved in deionized water to acquire a clear solution and the mole ratio of $\mathrm{Fe}^{3+}$ to $\mathrm{Ba}^{2+}$ was $12: 1$. The citric acid was dissolved into an aqueous solution and then mixed with the nitrate solution acquired above. The molar ratio of citric acid and total moles of $\mathrm{Fe}^{3+}$ and $\mathrm{Ba}^{2+}$ was fixed at $2: 1$. An ammonia solution was added into the mixed solution slowly while stirring until the $\mathrm{pH}$ reached 7 . The mixture was then placed in a water bath at $80{ }^{\circ} \mathrm{C}$ for $3 \mathrm{~h}$ and the temperature was increased to $120^{\circ} \mathrm{C}$ to evaporate the water. The prepared EG was added with continuous stirring until the mixture appeared as an oily gel and the mass fraction of EG was $15 \%$. The temperature was increased to $200{ }^{\circ} \mathrm{C}$, and the mixture was bubbled up and automatically ignited. After the combustion reaction, fluffy black powder was obtained. Finally, the fluffy black powder was calcined at $800{ }^{\circ} \mathrm{C}$ for $3 \mathrm{~h}$ to obtain the $\mathrm{G} / \mathrm{BF}$ sandwich structured nanocomposite. ${ }^{19}$ After the reaction, the black product was washed with $\mathrm{HCl}$ solution ( $2 \mathrm{M}$ ) several times to obtain graphene. When the molar ratio of $\mathrm{Fe}^{3+}, \mathrm{Ba}^{2+}$ and $\mathrm{La}^{3+}$ was $12: 0.8: 0.2$, the sandwich microstructured G/LBF nanocomposites could be obtained by a similar process.

\subsection{Structural characterizations and microwave absorption measurements}

For EMW parameter measurements, these nanocomposites were uniformly mixed with $75 \mathrm{wt} \%$ paraffin wax, and the compounds were poured into a planar rectangular mold and dried for about 24 hours in air. The prepared films were tailored precisely to the size of a rectangular waveguide $(22.86 \mathrm{~mm} \times$ $10.16 \mathrm{~mm}$ ) for EMW parameter tests.

The morphology and microstructure of these nanocomposites were characterized by a scanning electron microscope (SEM, FEINNS450, FEI) equipped with an energy dispersive spectrometer (EDS) and a transmission electron microscope (TEM, JEM-100CX11, FEI). Fourier transform infrared (FTIR) spectra were measured using Nicolet IS10 FTIR with $\mathrm{KBr}$. Ultraviolet-visible absorption (UV-vis) spectra were measured using Lambda 35. The conductivity of the samples was obtained by a four-probe conductivity tester (5601Y). Raman spectra of samples were measured using a Lab RAM HR confocal Raman system with $532 \mathrm{~nm}$ diode laser excitation at room temperature. X-ray diffraction (XRD) (X'Pert Pro, 
PANalytical, with $\mathrm{Cu} \mathrm{K} \alpha$ radiation) patterns were recorded to identify phase structure. The hysteresis loops of samples were measured using a vibrating sample magnetometer (VSM, Lake Shore 7410, Quantum Design). The reflection loss of these nanocomposites was measured by a vector network analyzer (VNA Agilent technologies E8362B; $10 \mathrm{MHz}-20 \mathrm{GHz}$ ).

\section{Results and discussion}

\subsection{Morphological characterization}

The SEM images of EG, $\mathrm{BaFe}_{12} \mathrm{O}_{19}, \mathrm{Ba}_{0.8} \mathrm{La}_{0.2} \mathrm{Fe}_{12} \mathrm{O}_{19}, \mathrm{G} / \mathrm{LBF}$ nanocomposite and graphene are shown in Fig. 2. Fig. 2(a) shows the microstructure of EG. It can be seen clearly that EG has a large slice spacing and there are several folds on the surface. The slice spacing is up to $2 \mu \mathrm{m}$ and each graphite sheet may consist of hundreds of graphene layers. Moreover, the large slice provides the opportunity for the oily gel to enter in the graphite sheet spacing. $\mathrm{BaFe}_{12} \mathrm{O}_{19}$ particles are shown in Fig. 2(b). The sizes of $\mathrm{BaFe}_{12} \mathrm{O}_{19}$ particles are different, and the particles undergo a certain degree of agglomeration. Fig. 2(c) shows the microstructure of $\mathrm{Ba}_{0.8} \mathrm{La}_{0.2} \mathrm{Fe}_{12} \mathrm{O}_{19}$ particles. Ladoped $\mathrm{BaFe}_{12} \mathrm{O}_{19}$ particles do not show any evident morphological changes. However, it should be noted that the dispersibility of La-doped particles becomes better. The sandwich microstructured G/LBF nanocomposite can be clearly seen in Fig. 2(d). $\mathrm{Ba}_{0.8} \mathrm{La}_{0.2} \mathrm{Fe}_{12} \mathrm{O}_{19}$ particles are located between graphene sheets, as shown in the labeled area. The surface of graphene exhibiting a transparent film has clear folds. $\mathrm{Ba}_{0.8^{-}}$ $\mathrm{La}_{0.2} \mathrm{Fe}_{12} \mathrm{O}_{19}$ particles enwrapped with graphene are easily identified, and the distribution of these particles between the graphene sheets is fairly uniform. Fig. 2(e) shows an image of the edge of the sandwiched structure. There are numerous $\mathrm{Ba}_{0.8} \mathrm{La}_{0.2} \mathrm{Fe}_{12} \mathrm{O}_{19}$ particles between the graphene sheets, and some particles are also distributed on the graphene surface. The edge of the sandwiched structure can be clearly seen in the labeled area. $\mathrm{Ba}_{0.8} \mathrm{La}_{0.2} \mathrm{Fe}_{12} \mathrm{O}_{19}$ particles are embedded in the gaps between the graphene sheets, indicating that the oily gel has entered into the graphite layers successfully and the secondary expansion process of EG is adequate. The clear morphology of graphene is shown in Fig. 2(f). It exhibits a very thin transparent shape and is wrapped with $\mathrm{Ba}_{0.8} \mathrm{La}_{0.2} \mathrm{Fe}_{12} \mathrm{O}_{19}$ particles. Moreover, it seems the formation of $\mathrm{Ba}_{0.8} \mathrm{La}_{0.2} \mathrm{Fe}_{12} \mathrm{O}_{19}$ particles has a significant secondary expansion effect on the graphite sheets. The volume increase effect of $\mathrm{Ba}_{0.8} \mathrm{La}_{0.2} \mathrm{Fe}_{12} \mathrm{O}_{19}$ between the graphene sheets leads to an evident bulge phenomenon. Fig. 2(g) and (h) represent pure graphene. Fig. 2(g) shows large translucent graphene with several folds. It is very thin and exhibits a flexible sheet with a large surface. In addition, it can be found that there are some impurities on the surface of graphene. This may be because some ferrite particles have not been removed. The clear folds of graphene are shown in Fig. 2(h). Its surface is quite clean and distributed with numerous evident folds. Fig. 2(i) is the EDS spectrum image of the rectangular area selected in Fig. 2(f). There are five main elements and the mass ratio of each element is shown on the upper right corner. This result is in relative agreement with the theoretical value.
TEM images of G/LBF nanocomposite are shown in Fig. 3. It is clear that graphene with several folds shows a translucent film in Fig. 3(a). $\mathrm{Ba}_{0.8} \mathrm{La}_{0.2} \mathrm{Fe}_{12} \mathrm{O}_{19}$ particles have a uniform size and show no apparent agglomeration. In addition, it should be noted that these particles have different color depth. The lightcolored particles are located between the sheets of graphene and the dark-colored ones are distributed on the graphene surface. Fig. 3(b) is the high magnification image of G/LBF nanocomposite. $\mathrm{Ba}_{0.8} \mathrm{La}_{0.2} \mathrm{Fe}_{12} \mathrm{O}_{19}$ particles with various sizes can be seen, and the mean size is about $100 \mathrm{~nm}$. Furthermore, it can be easily noted that some of these particles are located between graphene sheets, while a small amount them are on the graphene surface. It is clear that graphene with numerous folds shows a translucent film, as shown in Fig. 3(c). The structure exhibits two graphene sheets stacked together, and shows a large surface and very low thickness.

Fig. 4(a) shows the XRD patterns of graphene, $\mathrm{BaFe}_{12} \mathrm{O}_{19}$, $\mathrm{Ba}_{0.8} \mathrm{La}_{0.2} \mathrm{Fe}_{12} \mathrm{O}_{19}$, and $\mathrm{G} / \mathrm{BF}$ and $\mathrm{G} / \mathrm{LBF}$ nanocomposites. The main diffraction peaks of $\mathrm{BaFe}_{12} \mathrm{O}_{19}$ are observed at $2 \theta$ values of $31.4^{\circ}, 35.2^{\circ}, 42.3^{\circ}, 61.1^{\circ}$ and $76.3^{\circ}$ corresponding to the (110), (114), (205), (217) and (220) reflections, respectively. ${ }^{20}$ The observed peaks of $\mathrm{BaFe}_{12} \mathrm{O}_{19}$ are matched with the standard XRD pattern, indicating that $\mathrm{BaFe}_{12} \mathrm{O}_{19}$ is successfully synthesized by the auto-combustion method. The XRD pattern of $\mathrm{Ba}_{0.8} \mathrm{La}_{0.2} \mathrm{Fe}_{12} \mathrm{O}_{19}$ did not significantly change compared to the $\mathrm{BaFe}_{12} \mathrm{O}_{19}$ pattern. It means that La doping has no effect on the formation of $\mathrm{BaFe}_{12} \mathrm{O}_{19}$. Graphene exhibits a broad characteristic diffraction peak at $2 \theta=26.20^{\circ}$, which is typical of multilayered graphene, indicating that EG has been peeled into multilayered graphene during the self-combustion and swelling of $\mathrm{BaFe}_{12} \mathrm{O}_{19}$. The XRD patterns of $\mathrm{G} / \mathrm{BF}$ and $\mathrm{G} / \mathrm{LBF}$ are similar, and these patterns have a peak at $2 \theta=26.20^{\circ}$. EG is not completely converted to graphene during the auto-combustion process of $\mathrm{Ba}_{0.8} \mathrm{La}_{0.2} \mathrm{Fe}_{12} \mathrm{O}_{19}$. The peaks of $\mathrm{Ba}_{0.8} \mathrm{La}_{0.2} \mathrm{Fe}_{12} \mathrm{O}_{19}$ do not show a significant change in the $\mathrm{G} / \mathrm{LBF}$ pattern, indicating that there is no chemical reaction between $\mathrm{Ba}_{0.8} \mathrm{La}_{0.2} \mathrm{Fe}_{12} \mathrm{O}_{19}$ and EG. The FTIR spectra of graphene and G/LBF nanocomposite are shown in Fig. 4(b). The relative apparent peaks of graphene are $1578 \mathrm{~cm}^{-1}$ and $936 \mathrm{~cm}^{-1}$, and there are no other evident peaks. The peak at $1578 \mathrm{~cm}^{-1}$ is the characteristic peak of the $\mathrm{C}=\mathrm{C}$ bond in the $\mathrm{sp}^{2}$ structure of graphite, which means that there is a small amount of graphite in the graphene. ${ }^{21}$ This may be because the peeling of graphite is not complete. The characteristic peaks of G/LBF occur at 2930, 1578, 1450, 936 and $618 \mathrm{~cm}^{-1}$. The peak at $2930 \mathrm{~cm}^{-1}$ is attributed to the $\mathrm{C}-\mathrm{H}$ stretching vibration, the $\mathrm{C}-\mathrm{N}$ group is located at $1450 \mathrm{~cm}^{-1}$, and the peak at $2930 \mathrm{~cm}^{-1}$ represents some oxygen group. ${ }^{22}$ This may be due to the presence of some impurities after the combustion process of barium ferrite gel and the G/LBF nanocomposite is inorganic materials.

The crystal size of $\mathrm{Ba}_{0.8} \mathrm{La}_{0.2} \mathrm{Fe}_{12} \mathrm{O}_{19}$ particles can be calculated using the Debye-Scherrer formula: $D=k \lambda / \beta \cos \theta$, where $D$ is the average crystal size of particles, $k$ is the shape factor, $\lambda$ is the X-ray wavelength, $\theta$ is the half angle in degrees and $\beta$ is the full width at half maximum. $k$ is often assigned a value of 0.89 , which depends on several factors, including Miller index of the reflecting plane and shape of the crystal. ${ }^{23}$ 

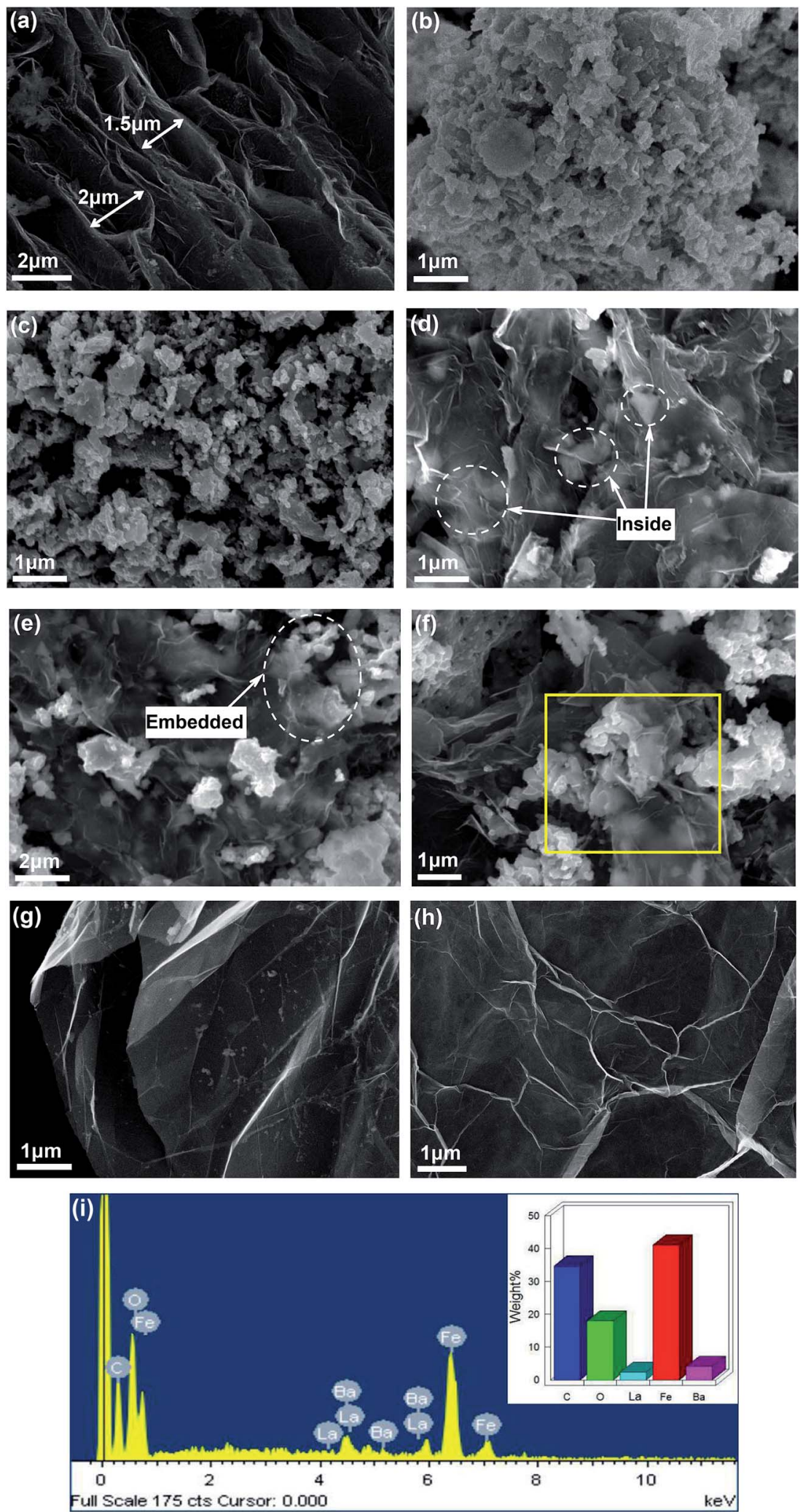

Fig. 2 SEM images of (a) $E G$, (b) $\mathrm{BaFe}_{12} \mathrm{O}_{19}$, (c) $\mathrm{Ba}_{0.8} \mathrm{La}_{0.2} \mathrm{Fe}_{12} \mathrm{O}_{19}$, (d-f) $\mathrm{G} / \mathrm{LBF}$ nanocomposite, (g-h) graphene and (i) EDS spectrum image of the rectangular area selected in (f).

The (217) reflection of the observed X-ray data is chosen for calculating crystal size of $\mathrm{Ba}_{0.8} \mathrm{La}_{0.2} \mathrm{Fe}_{12} \mathrm{O}_{19}$ particles. The average size of the $\mathrm{Ba}_{0.8} \mathrm{La}_{0.2} \mathrm{Fe}_{12} \mathrm{O}_{19}$ particles calculated using the abovementioned equation is about $96 \mathrm{~nm}$. It is in agreement with the average particle size as determined by TEM. 

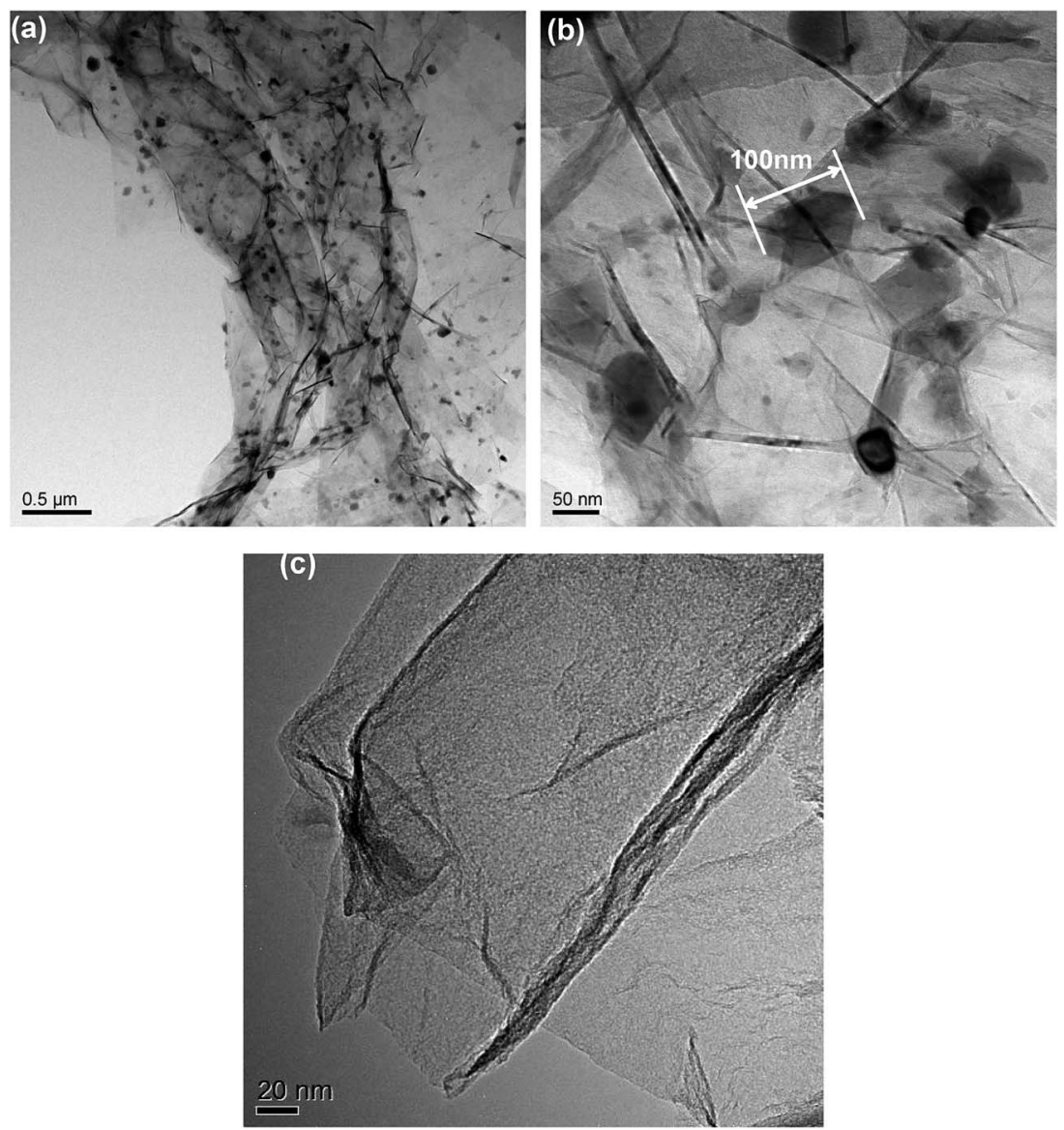

Fig. 3 TEM images of (a) G/LBF nanocomposite, (b) high magnification of G/LBF nanocomposite and (c) graphene.
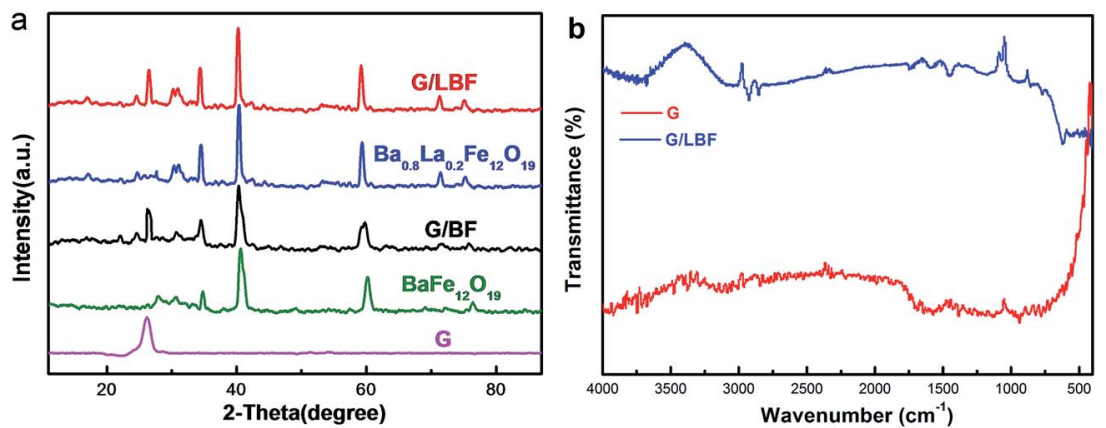

Fig. 4 (a) XRD patterns of graphene, $\mathrm{BaFe}_{12} \mathrm{O}_{19}, \mathrm{Ba}_{0.8} \mathrm{La}_{0.2} \mathrm{Fe}_{12} \mathrm{O}_{19}$, and $\mathrm{G} / \mathrm{BF}$ and $\mathrm{G} / \mathrm{LBF}$ nanocomposite and (b) FTIR spectra of graphene and $\mathrm{G} /$ LBF nanocomposites.

Fig. 5(a) shows the Raman spectra of the G/BF and G/LBF nanocomposites. The Raman spectra of these nanocomposites exhibit characteristic D, G and 2D bands at $1350 \mathrm{~cm}^{-1}, 1580$ $\mathrm{cm}^{-1}$ and $2680 \mathrm{~cm}^{-1}$, respectively. The $\mathrm{G}$ band is associated with $\mathrm{E}_{2 \mathrm{~g}}$ symmetry and is generated due to in-plane vibration of $\mathrm{sp}^{2}$ carbon atoms of graphene. The $\mathrm{D}$ band appears from a breathing mode of a $K$-point phonon of $\mathrm{A}_{1 \mathrm{~g}}$ symmetry and it implies the degree of graphene defects and irregularities. The 2D peak originates from the double-resonance Raman process of two phonons with opposite momentum. The intensity ratio of the $\mathrm{D}$ and $\mathrm{G}$ peaks $\left(I_{\mathrm{D}} / I_{\mathrm{G}}\right)$ has been used as a measure of ordered and disordered degree in graphene. ${ }^{24}$ It can be seen that the Raman spectra of G/BF and G/LBF are almost the same. The value of $I_{\mathrm{D}} / I_{\mathrm{G}}$ is quite high, indicating a highly disordered graphene. This is mainly because the auto-combustion process of $\mathrm{Ba}_{0.8} \mathrm{La}_{0.2} \mathrm{Fe}_{12} \mathrm{O}_{19}$ damages the microstructure of graphene to a certain degree. The peaks of $\mathrm{BaFe}_{12} \mathrm{O}_{19}$ are between $260 \mathrm{~cm}^{-1}$ and $700 \mathrm{~cm}^{-1}$, and they are similar to those of $\mathrm{Ba}_{0.8} \mathrm{La}_{0.2} \mathrm{Fe}_{12} \mathrm{O}_{19}$, as shown in Fig. 5(a). ${ }^{25}$ The optical properties of graphene and G/LBF are investigated using their UV-vis absorption spectra. As 

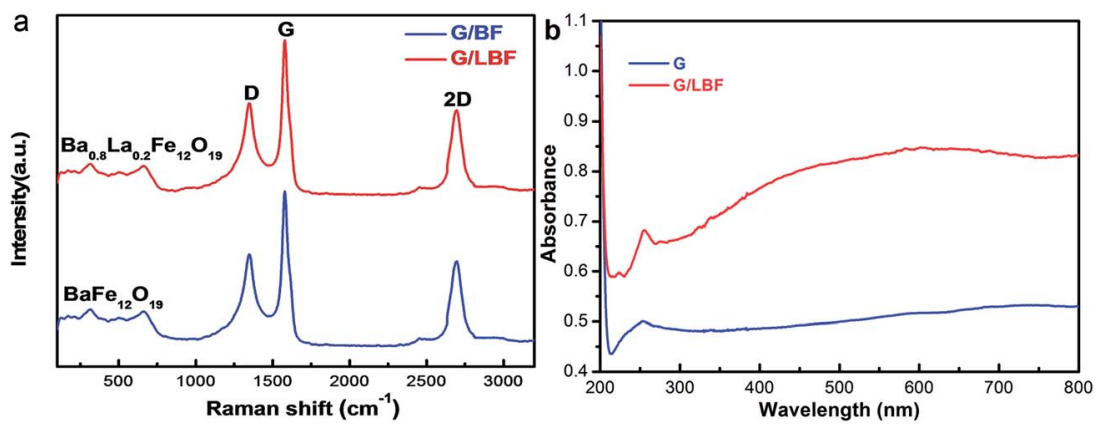

Fig. 5 (a) Raman spectra of G/BF and G/LBF nanocomposites and (b) UV-vis absorption spectra of graphene and G/LBF nanocomposite.

shown in Fig. 5(b), there is an absorption peak at a wavelength of $251 \mathrm{~nm}$, corresponding to the transition of aromatic C-C bonds. The small peak for G/LBF at $223 \mathrm{~nm}$ may be caused by some impurities..$^{26}$ In addition, it can be found that the G/LBF nanocomposite exhibits remarkable light absorption in the visible-light region.

\subsection{Magnetic properties}

The hysteresis loops of $\mathrm{BaFe}_{12} \mathrm{O}_{19}, \mathrm{G} / \mathrm{BF}$ nanocomposite, $\mathrm{Ba}_{0.8} \mathrm{La}_{0.2} \mathrm{Fe}_{12} \mathrm{O}_{19}$ and $\mathrm{G} / \mathrm{LBF}$ nanocomposite were measured at room temperature, and the results are shown in Fig. 6(a). When $H$ is infinite, the saturation magnetization $\left(M_{\mathrm{S}}\right)$ value can be obtained from the $M$ value. The coercive force $\left(H_{\mathrm{C}}\right)$ and residual magnetization values can be obtained directly from the hysteresis loops. ${ }^{27}$ The $M_{\mathrm{S}}$ value of $\mathrm{BaFe}_{12} \mathrm{O}_{19}$ is about $38.5 \mathrm{emu}$ $\mathrm{g}^{-1}$, while that of $\mathrm{Ba}_{0.8} \mathrm{La}_{0.2} \mathrm{Fe}_{12} \mathrm{O}_{19}$ is $30.68 \mathrm{emu} \mathrm{g}^{-1}$. It means that La doping reduces saturation magnetization. $\mathrm{G} / \mathrm{BF}$ has an $M_{\mathrm{S}}$ value of about $34.26 \mathrm{emu} \mathrm{g}^{-1}$, which is lower than that of pure $\mathrm{BaFe}_{12} \mathrm{O}_{19}$. The $M_{\mathrm{S}}$ value of $\mathrm{G} / \mathrm{LBF}$ is about $26.55 \mathrm{emu} \mathrm{g}^{-1}$, which is also lower than that of pure $\mathrm{Ba}_{0.8} \mathrm{La}_{0.2} \mathrm{Fe}_{12} \mathrm{O}_{19}$. This is mainly due to the fact that non-magnetic graphene reduced magnetic saturation strength. From the enlarged image on the upper left corner of Fig. 6(a), it can be deduced that the coercivity of G/LBF is about 188.76 Oe, and the high coercivity is beneficial to magnetic loss at a relatively low frequency. ${ }^{28}$ Fig. 6(b) shows the conductivity of G/LBF nanocomposites with different contents of graphene. It is clear that the conductivity of the G/LBF nanocomposites increases with graphene content. It is because graphene has excellent conductivity and the ferrite materials are weakly conductive. ${ }^{29}$

\subsection{Electromagnetic wave absorbing properties}

The complex permittivity $\left(\varepsilon^{\prime}, \varepsilon^{\prime \prime}\right)$ and complex permeability $\left(\mu^{\prime}, \mu^{\prime \prime}\right)$ spectra of graphene, $\mathrm{BaFe}_{12} \mathrm{O}_{19}, \mathrm{Ba}_{0.8} \mathrm{La}_{0.2} \mathrm{Fe}_{12} \mathrm{O}_{19}, \mathrm{G} / \mathrm{BF}$ and $\mathrm{G} / \mathrm{LBF}$ nanocomposites $v s$. frequency in $2-18 \mathrm{GHz}$ are shown in Fig. 7. The real part of complex permittivity and permeability is mainly associated with the amount of polarization occurring in the material and symbolizes the storage ability of the electric and magnetic energy, whereas the imaginary part accounts for dielectric and magnetic losses. ${ }^{30}$ The real part of complex permittivity spectra for different materials is shown in Fig. 7(a). Its overall trend decreases with increasing frequency. The value of graphene is the highest, because it has excellent electric conductivity. The spectra of $\mathrm{BaFe}_{12} \mathrm{O}_{19}$ and $\mathrm{Ba}_{0.8} \mathrm{La}_{0.2} \mathrm{Fe}_{12} \mathrm{O}_{19}$ are almost the same, and their values are very close. It illustrates that La doping does not have a significant effect on the dielectric real part. The spectra of G/BF and G/LBF are also similar, but their values are higher than those of pure $\mathrm{BaFe}_{12} \mathrm{O}_{19}$ and $\mathrm{Ba}_{0.8} \mathrm{La}_{0.2} \mathrm{Fe}_{12} \mathrm{O}_{19}$. It is mainly because ferrite conductivity in the latter is poor, and graphene improves the conductivity of these nanocomposites effectively. The imaginary part of complex permittivity spectra for different materials is shown in Fig. 7(b). The value of graphene is quite low, indicating poor dielectric loss. This is due to its bad impedance matching performance.
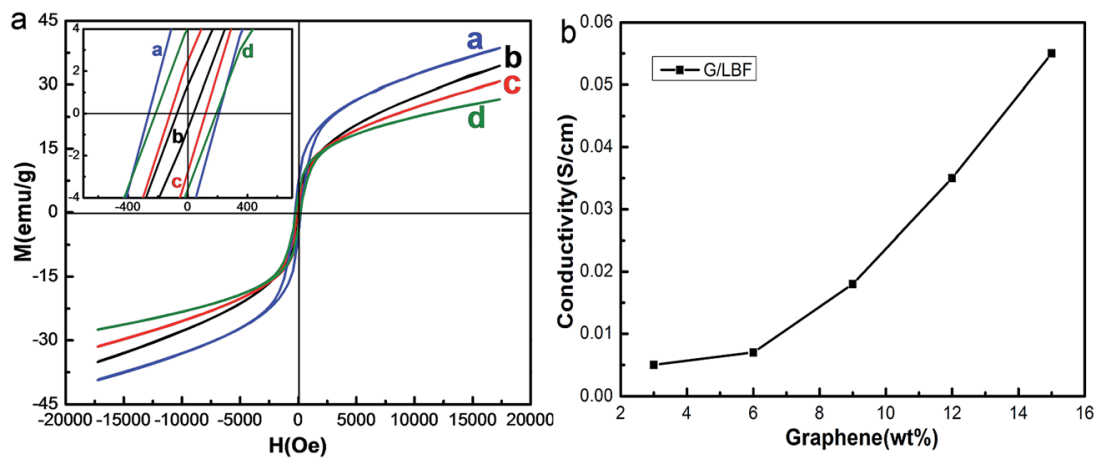

Fig. 6 (a) Hysteresis loops of (a) $\mathrm{BaFe}_{12} \mathrm{O}_{19}$, (b) $\mathrm{G} / \mathrm{BF}$, (c) $\mathrm{Ba}_{0.8} \mathrm{La}_{0.2} \mathrm{Fe}_{12} \mathrm{O}_{19}$ and (d) G/LBF nanocomposite and (b) conductivity of G/LBF nanocomposites with different contents of graphene. 

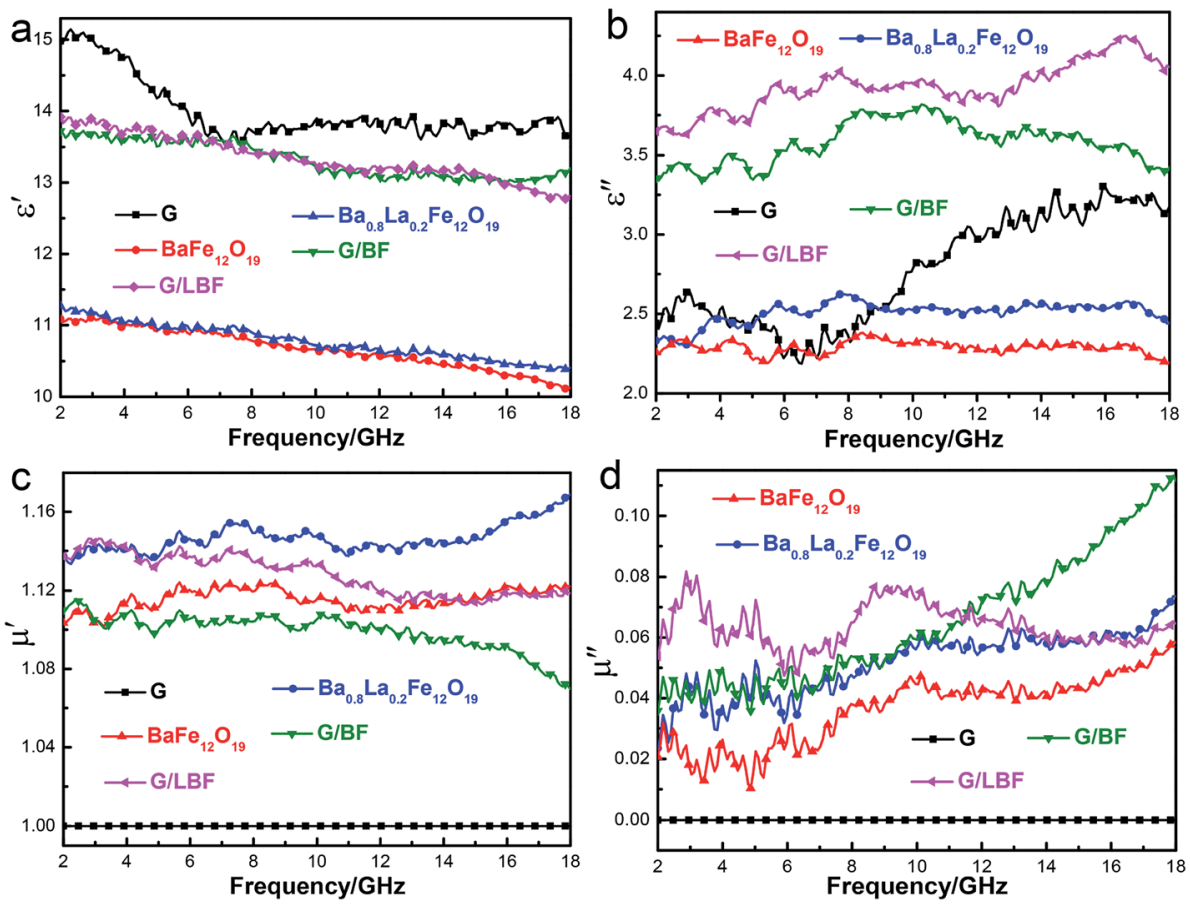

Fig. 7 (a-b) Complex permittivity $\left(\varepsilon^{\prime}, \varepsilon^{\prime \prime}\right)$ and $(c-d)$ complex permeability $\left(\mu^{\prime}, \mu^{\prime \prime}\right)$ spectra of graphene, $\mathrm{BaFe}_{12} \mathrm{O}_{19}, \mathrm{Ba}_{0.8} \mathrm{La}_{0.2} \mathrm{Fe}_{12} \mathrm{O}_{19}, \mathrm{G} / \mathrm{BF}$ and $\mathrm{G} /$ LBF nanocomposite vs. frequency within $2-18 \mathrm{GHz}$.

$\mathrm{Ba}_{0.8} \mathrm{La}_{0.2} \mathrm{Fe}_{12} \mathrm{O}_{19}$ shows higher values than $\mathrm{BaFe}_{12} \mathrm{O}_{19}$, and it illustrates that La doping improved the dielectric constant. It is mainly due to the special electronic structure of rare earth elements, which increases the interaction with the electromagnetic wave. The G/BF and G/LBF with high values mean that they have strong dielectric capacity. Fig. 7(c) and (d) show the complex permeability spectra for different materials. The real and imaginary parts of graphene are 1 and 0 , respectively, owing to the fact that graphene is non-magnetic. In Fig. 7(c), the real part value of $\mathrm{Ba}_{0.8} \mathrm{La}_{0.2} \mathrm{Fe}_{12} \mathrm{O}_{19}$ is higher than that of $\mathrm{BaFe}_{12} \mathrm{O}_{19}$, indicating that $\mathrm{La}$ doping improved magnetic property. Furthermore, a high real part value indicates good impedance matching and it facilitates more EMW entry into the composites. $^{31}$ In addition, it should be noted that the value of G/BF nanocomposite is lower than that of pure $\mathrm{BaFe}_{12} \mathrm{O}_{19}$ and the value of $\mathrm{G} / \mathrm{LBF}$ nanocomposite is lower than that of pure $\mathrm{Ba}_{0.8} \mathrm{La}_{0.2} \mathrm{Fe}_{12} \mathrm{O}_{19}$. This is because non-magnetic graphene occupies a certain proportion of the nanocomposites. The imaginary part of complex permeability is a measure of magnetic loss. Magnetic materials generate magnetic losses during the repeated magnetization and demagnetization process in the electromagnetic field and the magnetic loss intensity is related to EMW frequency. ${ }^{32}$ In Fig. $7(\mathrm{~d})$, $\mathrm{Ba}_{0.8} \mathrm{La}_{0.2} \mathrm{Fe}_{12} \mathrm{O}_{19}$ exhibits a higher value than $\mathrm{BaFe}_{12} \mathrm{O}_{19}$, indicating a higher magnetic loss. Moreover, it can be found that magnetic loss property improved after combining with graphene. This is owing to the good impedance matching performance of these nanocomposites. As the frequency increases, the imaginary part value of complex permeability of G/BF increases, while that of G/LBF is relatively steady.
The loss tangent is a measure of electromagnetic loss capability for an absorbing material and a large loss tangent is generally expected. Fig. 8 shows the dielectric constant tangent $\left(\tan \delta_{\varepsilon}=\varepsilon^{\prime \prime} / \varepsilon^{\prime}\right)$ and magnetic loss tangent $\left(\tan \delta_{\mu}=\mu^{\prime \prime} / \mu^{\prime}\right)$ spectra of graphene, $\mathrm{BaFe}_{12} \mathrm{O}_{19}, \mathrm{Ba}_{0.8} \mathrm{La}_{0.2} \mathrm{Fe}_{12} \mathrm{O}_{19}, \mathrm{G} / \mathrm{BF}$ and $\mathrm{G} / \mathrm{LBF}$ nanocomposites $v s$. frequency within the frequency of 2-18 GHz. It should be noted that $\tan \delta_{\varepsilon}$ is higher than $\tan \delta_{\mu}$ for the same material, regardless of frequency, indicating that reflection loss is mainly due to the dielectric constant. The $\tan \delta_{\varepsilon}$ of graphene is low, indicating poor EMW absorption performance. It may be due to the fact that impedance matching of pure graphene is poor. The tan $\delta_{\varepsilon}$ of G/LBF shows the highest value and its average value is about 0.30 , while that of $\mathrm{G} / \mathrm{BF}$ is only about 0.27, as shown in Fig. 8(a). Furthermore, $\mathrm{Ba}_{0.8} \mathrm{La}_{0.2} \mathrm{Fe}_{12} \mathrm{O}_{19}$ exhibits a higher $\tan \delta_{\varepsilon}$ than $\mathrm{BaFe}_{12} \mathrm{O}_{19}$, indicating that $\mathrm{La}$ doping improved the dielectric constant. The $\tan \delta_{\varepsilon}$ of G/LBF shows a significant increase compared with that of $\mathrm{Ba}_{0.8} \mathrm{La}_{0.2^{-}}$ $\mathrm{Fe}_{12} \mathrm{O}_{19}$, which means that G/LBF has better EMW absorption performance. This is mainly due to the excellent electrical conductivity of graphene and because the sandwiched microstructure of nanocomposite is conducive to multiple reflection of EMWs. In addition, good impedance matching of the nanocomposite is an important factor. In Fig. 8(b), it can be found that the spectra of $\tan \delta_{\mu}$ are similar to those of the imaginary part of complex permeability. The $\tan \delta_{\mu}$ of graphene is zero, indicating that it has no magnetic loss capability, because graphene is a non-magnetic material. The G/LBF composite has a higher value and is relatively steady. The magnetic loss capability of La-doped $\mathrm{BaFe}_{12} \mathrm{O}_{19}$ is improved, and it shows a better magnetic loss capability after combination with graphene. $^{33}$ 

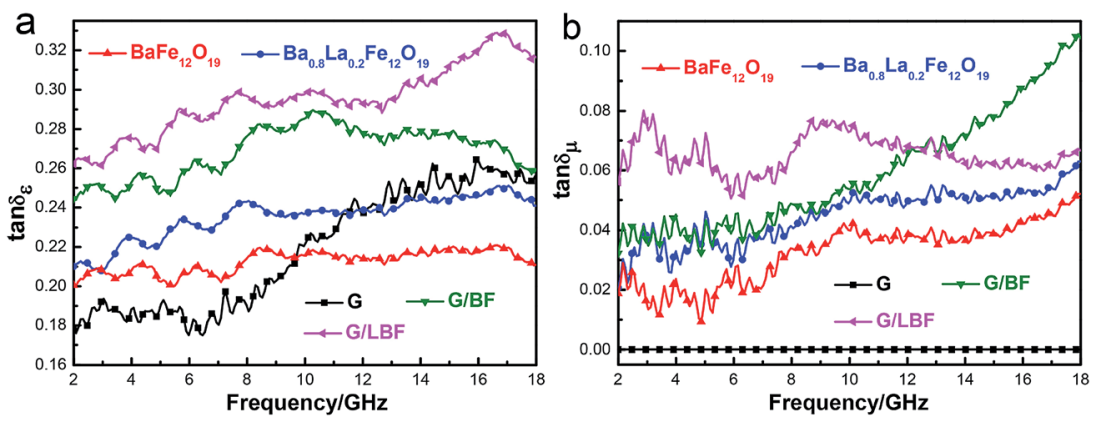

Fig. 8 (a) Dielectric constant tangent $\left(\tan \delta_{\varepsilon}\right.$ ) and (b) magnetic loss tangent ( $\tan \delta_{\mu}$ ) spectra of graphene, $\mathrm{BaFe}_{12} \mathrm{O}_{19}, \mathrm{Ba}_{0.8} \mathrm{La}_{0.2} \mathrm{Fe}_{12} \mathrm{O}_{19}, \mathrm{G} / \mathrm{BF}$ and G/LBF nanocomposites vs. frequency within 2-18 GHz.

Table 1 Influence of graphene mass ratio on electromagnetic wave absorbing properties of G/LBF nanocomposite with a thickness of 1 $\mathrm{mm}$

\begin{tabular}{llll}
\hline Mass ratio (wt\%) & $\begin{array}{l}\text { Range } \\
(\mathrm{dB})\end{array}$ & $\begin{array}{l}\text { Maximum reflection } \\
\text { loss }(\mathrm{dB})\end{array}$ & $\begin{array}{l}\text { Bandwidth } \\
(\mathrm{GHz})\end{array}$ \\
\hline 0 & $<-10$ & -14.60 & 1.88 \\
5 & $<-10$ & -19.13 & 2.27 \\
10 & $<-10$ & -32.22 & 2.93 \\
15 & $<-10$ & -40.26 & 3.87 \\
20 & $<-10$ & -37.80 & 3.25
\end{tabular}

Table 1 shows the influence of graphene mass ratio on the electromagnetic wave absorbing properties of G/LBF nanocomposites with a thickness of $1 \mathrm{~mm}$. It can be seen that EMW absorption performance of the G/LBF nanocomposites first increases and then decrease as graphene content increases. When the graphene mass ratio is $15 \%$, it shows the best absorbing properties with a maximum value of $-40.26 \mathrm{~dB}$. But when the mass ratio of graphene reaches $20 \%$, its performance is reduced. This may be because good impedance matching of the G/LBF nanocomposite is important and too much graphene is detrimental to its impedance matching performance. ${ }^{34}$

For a single-layer absorber, according to the transmission line theory, the calculation formulae are given as follows:

$$
\begin{gathered}
R(\mathrm{~dB})=20 \log _{10}\left|\frac{Z_{\text {in }}-1}{Z_{\text {in }}+1}\right| \\
Z_{\text {in }}=\left(\frac{\mu_{\mathrm{r}}}{\varepsilon_{\mathrm{r}}}\right)^{\frac{1}{2}} \tanh \left[\mathrm{j}\left(\frac{2 \pi f d}{c}\right)\left(\mu_{\mathrm{r}} \varepsilon_{\mathrm{r}}\right)^{\frac{1}{2}}\right]
\end{gathered}
$$

where $Z_{\text {in }}$ is the normalized input impedance at the free space and material interface, $\varepsilon_{\mathrm{r}}=\varepsilon-\mathrm{j} \varepsilon^{\prime \prime}$ is the complex permittivity of the absorber, $\mu_{\mathrm{r}}=\mu^{\prime}-\mathrm{j} \mu^{\prime \prime}$ is the complex permeability of the absorber, $f$ is the frequency of the microwaves in free space, $d$ is the thickness of the absorber, and $c$ is the velocity of light in free space. ${ }^{35}$ The reflection loss curves of different samples with same thickness $(2 \mathrm{~mm})$ are shown in Fig. 9(a). The reflection losses of graphene, $\mathrm{BaFe}_{12} \mathrm{O}_{19}$ and $\mathrm{Ba}_{0.8} \mathrm{La}_{0.2} \mathrm{Fe}_{12} \mathrm{O}_{19}$ are poor, while $\mathrm{G} / \mathrm{BF}$ and $\mathrm{G} / \mathrm{LBF}$ exhibit good reflection loss. As can be seen from the curves, the reflection loss curves of $\mathrm{G} / \mathrm{BF}$ and $\mathrm{G} /$
LBF both show broad peaks with a maximum of $-26.1 \mathrm{~dB}$ at $8.2 \mathrm{GHz}$ and $-38.61 \mathrm{~dB}$ at $8.1 \mathrm{GHz}$, respectively. The value of reflection loss below $-10 \mathrm{~dB}$ (absorbance is $90 \%$ of the wave) for G/LBF nanocomposite is up to $2.16 \mathrm{GHz}$. La doping improved EMW absorption performance effectively, and these nanocomposites have better performance than pure $\mathrm{BaFe}_{12} \mathrm{O}_{19}$ and $\mathrm{Ba}_{0.8} \mathrm{La}_{0.2} \mathrm{Fe}_{12} \mathrm{O}_{19}$. This is probably because the dielectric constant of graphene and the magnetic loss of ferrite are complementary. ${ }^{36}$ Graphene is a dielectric medium and ferrite is a magnetic medium. Three-dimensional images of reflection loss for different samples are shown in Fig. 9(c); from these figures, it is clear to see that G/LBF shows the best EMW absorption performance. The performance of the absorber mainly depends on two factors: the impedance matching at the interface and the complementarity between dielectric constant and magnetic loss. For a good matching of impedance, suitable electrical conductivity for the absorber is required. Both high and low conductivity results in bad microwave absorption due to skin effect and poor current thermal effect, respectively. ${ }^{37} \mathrm{G} /$ LBF shows excellent EMW absorption performance above, and in order to determine the thickness effect of samples on EMW absorption performance, reflection loss of the G/LBF nanocomposite with different thicknesses of 1-3 $\mathrm{mm}$ are calculated. The reflection loss curves are shown in Fig. 9(b) and the intuitive three-dimensional images of reflection loss are shown in Fig. 9(d). It demonstrates that the maximum reflection loss moves towards a lower frequency as thickness increases. This may be due to the different travel path of incident microwave in the absorber with different thickness and the synergistic effect of microwave absorption. The absorption frequency range can be adjusted according to requirement by altering the thickness of samples. ${ }^{38}$ For potential absorbers, large absorption bandwidth is expected. In this study, the sandwich microstructured G/LBF nanocomposite with a thickness of $1 \mathrm{~mm}$ shows the maximum reflection loss of $-40.26 \mathrm{~dB}$ at $14.9 \mathrm{GHz}$. Moreover, it should be noted that the value of reflection loss below $-10 \mathrm{~dB}$ could reach to $3.87 \mathrm{GHz}$. The sandwich microstructured G/LBF nanocomposite exhibits excellent EMW absorption performance and has important potential applications.

The experimental results mentioned above illustrate that the G/LBF composite has good impedance matching and complementarity between dielectric constant and magnetic loss. The 

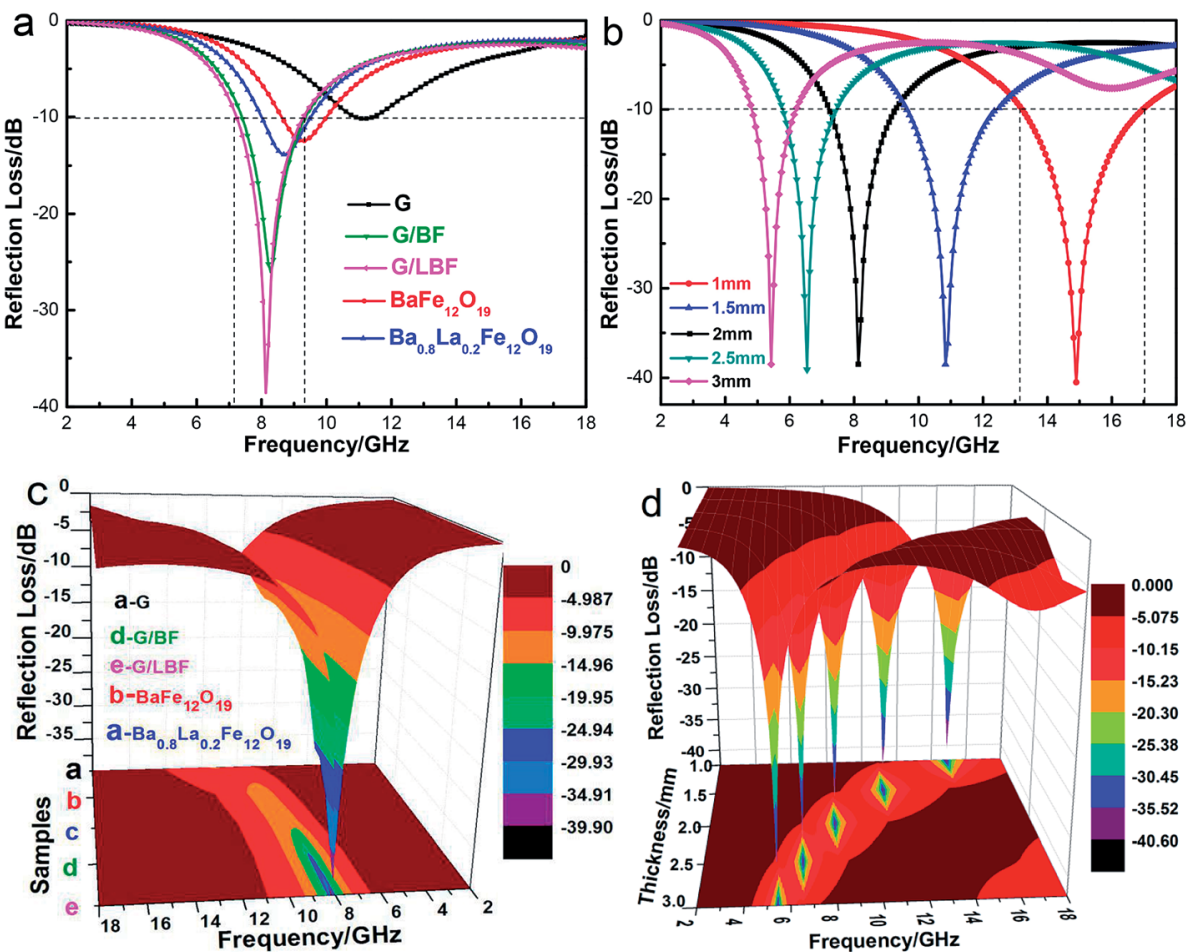

Fig. 9 (a) and (c) Reflection loss curves of graphene, $\mathrm{BaFe}_{12} \mathrm{O}_{19}, \mathrm{Ba}_{0.8} \mathrm{La}_{0.2} \mathrm{Fe}_{12} \mathrm{O}_{19}, \mathrm{G} / \mathrm{BF}$, and $\mathrm{G} / \mathrm{LBF}$ nanocomposite vs. frequency within $2-18$ $\mathrm{GHz}$; (b) and (d) reflection loss curves of G/LBF nanocomposite with different thickness vs. frequency within 2-18 GHz.

schematic representation of EMW absorption mechanism in the G/LBF nanocomposite is shown in Fig. $10 .^{39}$ The incident EMWs have several types of interactions in the nanocomposites. In addition to the excellent conductivity of graphene to increase the dielectric constant, $\mathrm{Ba}_{0.8} \mathrm{La}_{0.2} \mathrm{Fe}_{12} \mathrm{O}_{19}$ has good magnetism to increase the magnetic loss. Moreover, the sandwich structure is conducive to high losses of electromagnetic energy. It can be found that EMWs pass multiple reflections not only between the graphene sheets but also between the individual sandwiched microstructures of G/LBF. This process prolongs the propagation path of EMWs in the absorbers, and the multiple reflections of the microwave lead to high losses of electromagnetic energy. It is because the interaction of microwaves with dielectric materials intensifies molecular motions such as ionic conduction, bipolar polarization and relaxation. In addition, $\mathrm{Ba}_{0.8} \mathrm{La}_{0.2} \mathrm{Fe}_{12} \mathrm{O}_{19}$

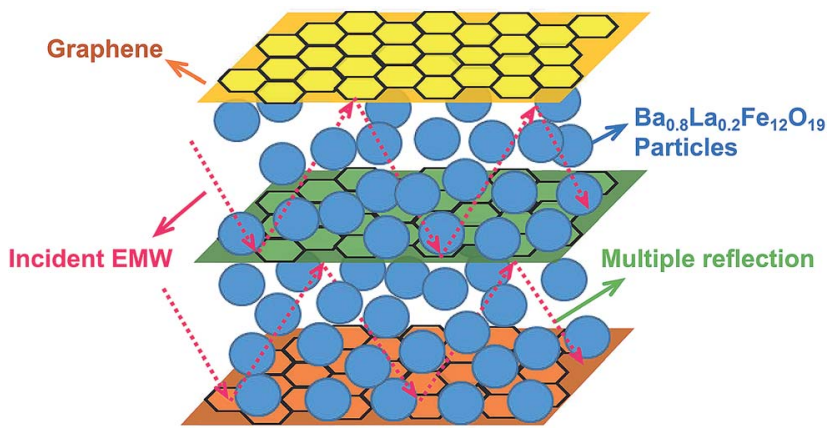

Fig. 10 Schematic of the EMW absorption mechanism of G/LBF nanocomposite. particles between graphene sheets can consume magnetic loss effectively. The multiple reflections between graphene sheets are conducive for more microwaves to go through the ferrite particles and thus increase magnetic losses. Therefore, the construction of sandwich microstructured $\mathrm{G} / \mathrm{LBF}$ is beneficial to increase electromagnetic performance.

\section{Conclusions}

The sandwich microstructured G/BF nanocomposite was successfully prepared through an in situ auto-combustion method and it showed better EMW absorbing properties compared to pure $\mathrm{BaFe}_{12} \mathrm{O}_{19}$. In addition, we doped $\mathrm{BaFe}_{12} \mathrm{O}_{19}$ with La and prepared the sandwich microstructured G/LBF. The sandwich microstructured G/LBF nanocomposite exhibited a saturation magnetization of $26.55 \mathrm{emu} \mathrm{g}^{-1}$ at room temperature. The maximum reflection loss of the sandwich microstructured G/LBF with a thickness of $1 \mathrm{~mm}$ could reach up to $-40.26 \mathrm{~dB}$ and the frequency bandwidth value below $-10 \mathrm{~dB}$ was up to $3.87 \mathrm{GHz}$ in the frequency range of $2-18 \mathrm{GHz}$. The sandwich microstructured G/LBF may have extensive applications in military and electromagnetic pollution governance for its excellent EMW absorbing performance.

\section{Acknowledgements}

This study was supported by the Natural Science Foundation of China (51572221, 51672221), the China Aeronautical Science Fund (2014ZF53074), the Natural Science Foundation of 
Shaanxi Province (2016JQ5108) and sponsored by the Seed Foundation of Innovation and Creation for Graduate Students in Northwestern Polytechnical University (Z2017011).

\section{References}

1 W. Li, J. Wei, W. Wang, D. Hu, Y. Li and J. Guan, Mater. Des., 2016, 110, 27-34.

2 Y. Orikasa, T. Ina, T. Nakao, A. Mineshige, K. Amezawa, M. Oishi, H. Arai, Z. Ogumi and Y. Uchimoto, J. Phys. Chem. C, 2011, 33, 115-117.

3 Y. Liu, Z. Zhang, S. Xiao, C. Qiang, L. Tian and J. Xu, Appl. Surf. Sci., 2011, 257, 7678-7683.

4 Y. Zhu, M. D. Stoller, W. Cai, A. Velamakanni, R. D. Piner,

D. Chen and R. S. Ruoff, ACS Nano, 2015, 4, 1227-1233.

5 X. Huang, Z. Yin, S. Wu, X. Qi, Q. He, Q. Zhang, Q. Yan, F. Boey and H. Zhang, Small, 2011, 7, 1876-1902.

6 F. Ren, H. Yu, L. Wang, M. Saleem, Z. Tian and P. Ren, RSC Adv., 2014, 4, 14419-14431.

7 C. Wang, X. Han, P. Xu, X. Zhang, Y. Du, S. Hu, J. Wang and X. Wang, Appl. Phys. Lett., 2011, 98, 072906.

8 J. Zhao, Y. Xie, D. Guan, H. Hua, R. Zhong, Y. Qin, J. Fang, H. Liu and J. Chen, Sci. Rep., 2015, 5, 12544.

9 D. A. Vinnik, D. A. Zherebtsov, L. S. Mashkovtseva, S. Nemrava, A. S. Semisalova, D. M. Galimov, S. A. Gudkova, I. V. Chumanov, L. Isaenko and R. Niewa, J. Alloys Compd., 2015, 628, 480-484.

10 P. Bhattacharya, S. Dhibar, G. Hatui, A. Mandal, T. Das and C. K. Das, RSC Adv., 2014, 4, 17039-17053.

11 L. Kong, X. Yin, Y. Zhang, X. Yuan, Q. Li, F. Ye, L. Cheng and L. Zhang, J. Phys. Chem. C, 2013, 117, 19701-19711.

12 P. Avouris and C. Dimitrakopoulos, Mater. Today, 2012, 15, 86-97.

13 Z. Durmus, A. Durmus and H. Kavas, J. Mater. Sci., 2015, 50, 1201-1213.

14 M. Maddahfar, M. Ramezani and S. M. HosseinpourMashkani, Appl. Phys. A, 2016, 122, 1-9.

15 T. Wang, Y. Li, L. Wang, C. Liu, S. Geng, X. Jia, F. Yang, L. Zhang, L. Liu and B. You, RSC Adv., 2015, 5, 60114-60120.

16 F. Ren, G. Zhu, P. Ren, K. Wang, X. Cui and X. Yan, Appl. Surf. Sci., 2015, 351, 40-47.

17 J. Zhao, Y. Xie, M. Li, F. Xu, Z. Le, Y. Qin, D. Zhou, Z. Wang, H. Xu and J. Pan, Compos. Sci. Technol., 2014, 99, 147-153.

18 S. R. Dhakate, N. Chauhan, S. Sharma, J. Tawale, S. Singh, P. D. Sahare and R. B. Mathur, Carbon, 2011, 49, 1946-1954.
19 A. Ohlan, K. Singh, A. Chandra and S. K. Dhawan, ACS Appl. Mater. Interfaces, 2010, 2, 927-933.

20 J. Zhang, J. Fu, F. Li, E. Xie, D. Xue, N. J. Mellors and Y. Peng, ACS Nano, 2012, 6, 2273-2281.

21 Y. Xie, X. Hong, C. Yu, M. Li, Y. Huang, Y. Gao, J. Zhao, S. Yan, L. Shi and K. Zhang, Compos. Sci. Technol., 2013, 77, 8-13.

22 Y. Xie, J. Zhao, Z. Le, M. Li, J. Chen, Y. Gao, Y. Huang, Y. Qin, R. Zhong and D. Zhou, Compos. Sci. Technol., 2014, 99, 141146.

23 P. Anithakumari, B. P. Mandal, E. Abdelhamid, R. Naik and A. K. Tyagi, RSC Adv., 2016, 6, 16073-16080.

24 R. Saito, M. Hofmann, G. Dresselhaus, A. Jorio and M. S. Dresselhaus, Adv. Phys., 2011, 60, 413-550.

25 Y. Fu, Y. Wan, H. Xia and X. Wang, J. Power Sources, 2012, 213, 338-342.

26 Z. Hu, S. Lua, X. Yan and T. Lim, RSC Adv., 2015, 5, 8689186900.

27 C. Li, B. Huang and J. Wang, J. Mater. Sci., 2013, 48, 17021710.

28 F. Mazaleyrat, A. Pasko, A. Bartok and M. Lobue, J. Appl. Phys., 2011, 109, 331-333.

29 X. Hong, Y. Xie, X. Wang, M. Li, Z. Le, Y. Gao, Y. Huang, Y. Qin and Y. Ling, Compos. Sci. Technol., 2015, 117, 215-224.

30 B. Zhong, T. Sai, L. Xia, Y. Yu and G. Wen, Mater. Des., 2017, 121, 185-193.

31 Y. Xie, X. Hong, X. Wang, J. Zhao, Y. Gao, Y. Ling, S. Yan, L. Shi and K. Zhang, Synth. Met., 2012, 162, 1643-1647.

32 Q. Li, J. Pang, B. Wang, D. Tao, X. Xu, S. Li and J. Zhai, Adv. Powder Technol., 2013, 24, 288-294.

33 S. Kumar, R. R. Nair, P. B. Pillai, S. N. Gupta, M. A. Iyengar and A. K. Sood, ACS Appl. Mater. Interfaces, 2014, 6, 1742617436.

34 J. Zhao, J. Yu, Y. Xie, Z. Le, X. Hong, S. Ci, J. Chen, X. Qing, W. Xie and Z. Wen, Sci. Rep., 2016, 6, 20496.

35 X. Ren, H. Fan and Y. Cheng, Appl. Phys. A, 2016, 122, 1-7.

36 D. Sarkar, A. Bhattacharya, P. Nandy and S. Das, Mater. Lett., 2014, 1, 259-262.

37 Y. Sun, F. Xiao, X. Liu, C. Feng and C. Jin, RSC Adv., 2013, 3, 22554-22559.

38 F. Qin and C. Brosseau, J. Appl. Phys., 2012, 111, 6130161324.

39 N. Yousefi, X. Sun, X. Lin, X. Shen, J. Jia, B. Zhang, B. Tang, M. Chan and J. K. Kim, Adv. Mater., 2014, 26, 5480-5487. 